\title{
Production of hydroxycinnamoyl-shikimates and chlorogenic acid in Escherichia coli: production of hydroxycinnamic acid conjugates
}

Bong-Gyu Kim ${ }^{\dagger}$, Woo Dam Jung ${ }^{\dagger}$, Hyejung Mok and Joong-Hoon Ahn ${ }^{*}$

\begin{abstract}
Background: Hydroxycinnamates (HCS) are mainly produced in plants. Caffeic acid (CA), p-coumaric acid (PA), ferulic acid (FA) and sinapic acid (SA) are members of the HC family. The consumption of HC by human might prevent cardiovascular disease and some types of cancer. The solubility of HCs is increased through thioester conjugation to various compounds such as quinic acid, shikimic acid, malic acid, anthranilic acid, and glycerol. Although hydroxycinnamate conjugates can be obtained from diverse plant sources such as coffee, tomato, potato, apple, and sweet potato, some parts of the world have limited availability to these compounds. Thus, there is growing interest in producing $\mathrm{HC}$ conjugates as nutraceutical supplements.

Results: Hydroxycinnamoyl transferases (HCTs) including hydroxycinnamate-CoA shikimate transferase (HST) and hydroxycinnamate-CoA quinate transferase ( $\mathrm{HQT})$ were co-expressed with 4-coumarateCoA:ligase $(4 \mathrm{CL})$ in Escherichia coli cultured in media supplemented with HCs. Two hydroxycinnamoyl conjugates, $p$-coumaroyl shikimates and chlorogenic acid, were thereby synthesized. Total $29.1 \mathrm{mg} / \mathrm{L}$ of four different $p$-coumaroyl shikimates (3-p-coumaroyl shikimate, 4-p-coumaroyl shikimate, 3,4-di-p-coumaroyl shikimate, 3,5-di-p-coumaroyl shikimate, and 4,5-di-p-coumaroyl shikimate) was obtained and $16 \mathrm{mg} / \mathrm{L}$ of chlorogenic acid was synthesized in the wild type E. coli strain. To increase the concentration of endogenous acceptor substrates such as shikimate and quinate, the shikimate pathway in E. coli was engineered. A E. coli aroL and aroK gene were mutated and the resulting mutants were used for the production of $p$-coumaroyl shikimate. An E. coli aroD mutant was used for the production of chlorogenic acid. We also optimized the vector and cell concentration optimization.

Conclusions: To produce p-coumaroyl-shikimates and chlorogenic acid in E. coli, several E. coli mutants (an aroD mutant for chlorogenic acid production; an aroL, aroK, and aroKL mutant for $p$-coumaroyl-shikimates production) were made and each mutant was tested using an optimized construct. Using this strategy, we produced $235 \mathrm{mg} / \mathrm{L}$ of $p$-coumaroyl-shikimates and $450 \mathrm{mg} / \mathrm{L}$ of chlorogenic acid.
\end{abstract}

Keywords: Chlorogenic acid, Hydroxycinnamic acid, Hydroxycinnamate-CoA quinate transferase, Hydroxycinnamate-CoA shikimate transferase

\section{Background}

Hydroxycinnamates (HCs) such as caffeic acid (CA), $p$-coumaric acid (PA), ferulic acid (FA) and sinapic acid (SA) are mainly produced in plants. Because HCs are anti-oxidants that can scavenge free radicals, it has been suggested that consumption of HCs might prevent cardiovascular disease and some types of cancer. In

\footnotetext{
* Correspondence: jhahn@konkuk.ac.kr

${ }^{\dagger}$ Equal contributors

Department of Bioscience and Biotechnology, Bio/Molecular Informatics Center, Konkuk University, Seoul 143-701, Republic of Korea
}

addition, several biological functions of $\mathrm{HCs}$ have been described, such as protection against side effects of chemotheraphy and anti-osteoclast activity [1].

In plants, $\mathrm{HCs}$ are usually conjugated with other compounds such as quinic acid, shikimic acid, malic acid, anthranilic acid, and glycerol [2]. Chlorogenic acid, the conjugate of $\mathrm{CA}$ and quinate, is abundant in coffee, fruits, and vegetables, and is the primary source of $\mathrm{CA}$ in the human diet [3]. For regular consumers of coffee in Western countries, the major dietary phenolics are chlorogenic acids [4].

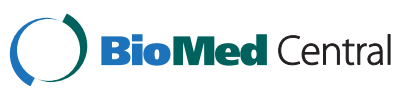


The solubility of HCs is increased through thioester conjugation to various compounds. Although there is growing evidence for their beneficial health effects, the fruits and vegetables that contain high levels of $\mathrm{HC}$ conjugates are of limited availability in some parts of the world. Thus, there is growing interest in producing $\mathrm{HC}$ conjugates as nutraceutical supplements. The primary strategy for obtaining diverse phytochemicals is by extracting them from plants. However, this approach faces several obstacles such as a limited availability of plant materials and high costs of extraction and purification. Alternatively, they can be produced in microorganisms. Phytochemicals such as flavonoids and stilbenes have been produced using microorganisms such as Escherichia coli and Saccharomyces cerevisiae [5-7].

Biosynthesis of $\mathrm{HC}$ conjugates is mediated by hydroxycinnamoyl transferases (HCTs), which use the coenzyme A thioester of $\mathrm{HCs}$ as a donor and various compounds such as quinate, and shikimate as $\mathrm{HC}$ acceptors. The formation of coenzyme A thioester with $\mathrm{HC}$ is catalyzed by 4-coumaric acid: CoA ligase (4-CL) [8]. Thus, genes encoding 4-CL and $\mathrm{HCT}$, are essential for producing $\mathrm{HC}$ conjugates from $\mathrm{HC}$. HCTs from several plants have been characterized [8-12]. HCTs show specificity for not only the acyl group donor but also the acyl group acceptor. When the acyl group acceptors are shikimate and quinate, the resulting conjugates are $p$-coumaroyl-O-shikimate and chlorogenic acid, respectively $[13,14]$.

E. coli is a good system for producing plant secondary metabolites including phytochemicals produced through the phenylpropanoid pathway [15]. We attempted to produce $\mathrm{HC}$ conjugates using $E$. coli. Shikimate and 3-dehydroquinate, which are acyl group acceptors for hydroxycinnamate-CoA shikimate transferases (HST) and hydroxycinnamate-CoA quinate transferases (HQT), respectively, can be synthesized through the shikimate pathway of E. coli [16,17]. Thus, it should be possible to alter the shikimate pathway to increase the concentrations of shikimate and quinate. However, the acyl donor (PA or CA) is a unique product of plants [18]. In order to produce $\mathrm{HCs}$ in $E$. coli, coexpression of several genes in $E$. coli is necessary, which leads to metabolic load. Therefore addition of exogenous HCs may circumvent this problem. In this report, we introduced either HST and $4 \mathrm{CL}$ (for the production of CA-shikimate), or HQT and 4CL (for the production of chlorogenic acid) into $E$. coli mutants that accumulate either shikimate or 3-hydroquinate. When the engineered cells were fed exogenous $\mathrm{HCs}$, a high yield of $\mathrm{HC}$ shikimate and $\mathrm{HC}$-quinate were obtained.

\section{Results}

Construction and selection of the optimum expression vector for the synthesis of $\mathrm{HC}$-shikimate

HC-shikimate was synthesized from HC-CoA and shikimate using HST (Figure 1). Two genes, HST from Nicotiana tabacum (NtHST) and 4CL from Oryza sativa $(O s 4 C L)$, were subcloned into an $E$. coli expression vector. Os4CL converts the $\mathrm{HCs}$ into the corresponding HC-CoAs. Os4CL has a better catalytic efficiency than other 4CLs [19]. NtHST uses HC-CoAs such as p-coumaroyl-CoA, caffeoyl-CoA, and feruloyl-CoA as acyl donors and shikimate as an acyl acceptor to produce HC-shikimate. NtHST was the first characterized HST and only a few number of HST has been biochemically characterized. NtHST also uses quinate as an acyl acceptor [9]. Both genes (NtHST and Os4CL) were subcloned into three different E. coli expression vectors, each of which has a different copy number in E. coli. Each construct (pA-NtHST-Os4CL, pC-NtHST-Os4CL, or pE-NtHST-Os4CL; see Table 1) was transformed into E. coli BL21(DE3) cells, and tested for its ability to produce $\mathrm{HC}$-shikimate when incubated with exogenous PA. As a control, the empty vector was transformed into E. coli. HCs including PA can enter into E. coli via the 4hydroxyphenylacetate permease (HpaX transporter) [20] and small phenolic compounds can be exported from E. coli into the culture medium. The transformant harboring pC-NtHST-Os4CL produced the highest concentration of $\mathrm{HC}$-shikimate derivatives $(29.1 \mathrm{mg} / \mathrm{L})$, whereas the titers of $\mathrm{HC}$-shikimate derivatives produced by the transformants harboring $\mathrm{pA}-\mathrm{NtHST}-\mathrm{Os} 4 \mathrm{CL}$ or $\mathrm{pE}-$ NtHST-Os4CL were 24.4 and $27.4 \mathrm{mg} / \mathrm{L}$, respectively. However, only PA was observed in the transformant harboring the empty vector. According to ANOVA test, a significant difference in PA-shikimates production at $\mathrm{P}=0.01$ was shown between $\mathrm{pC}-\mathrm{NtHST}-\mathrm{Os} 4 \mathrm{CL}$ and pA-NtHST-Os4CL or between pE-NtHST-Os4CL and pA-NtHST-Os4CL, but not between pC-NtHST-Os4CL and pE-NtHST-Os4CL. The construct present in the $\mathrm{pCDF}$ vector ( $\mathrm{pC}-\mathrm{NtHST}-\mathrm{Os} 4 \mathrm{CL}$ ) was used in further studies because this construct has a lower copy number than pE-NtHST-Os4CL. Therefore, pC-NtHST-Os4CL is expected to impose a lower metabolic load on E. coli.

Although each vector exhibited varying biotransformation efficiencies, they all had the same reaction profiles. As shown in Figure 2, four new peaks appeared (P1 P4). The molecular mass of P1 was $320.2 \mathrm{Da}$, indicating that one molecule of PA was attached to shikimate probably at the 5'-hydroxyl group of shikimate according to enzymatic studies $[9,10]$. In contrast, the molecular mass of the other three reaction products (P2 - P4) was 466.2 Da. Because the molecular mass of PA is $164 \mathrm{Da}$ and that of shikimate is $174 \mathrm{Da}$, the three reaction products must be conjugates of two PA molecules and one 


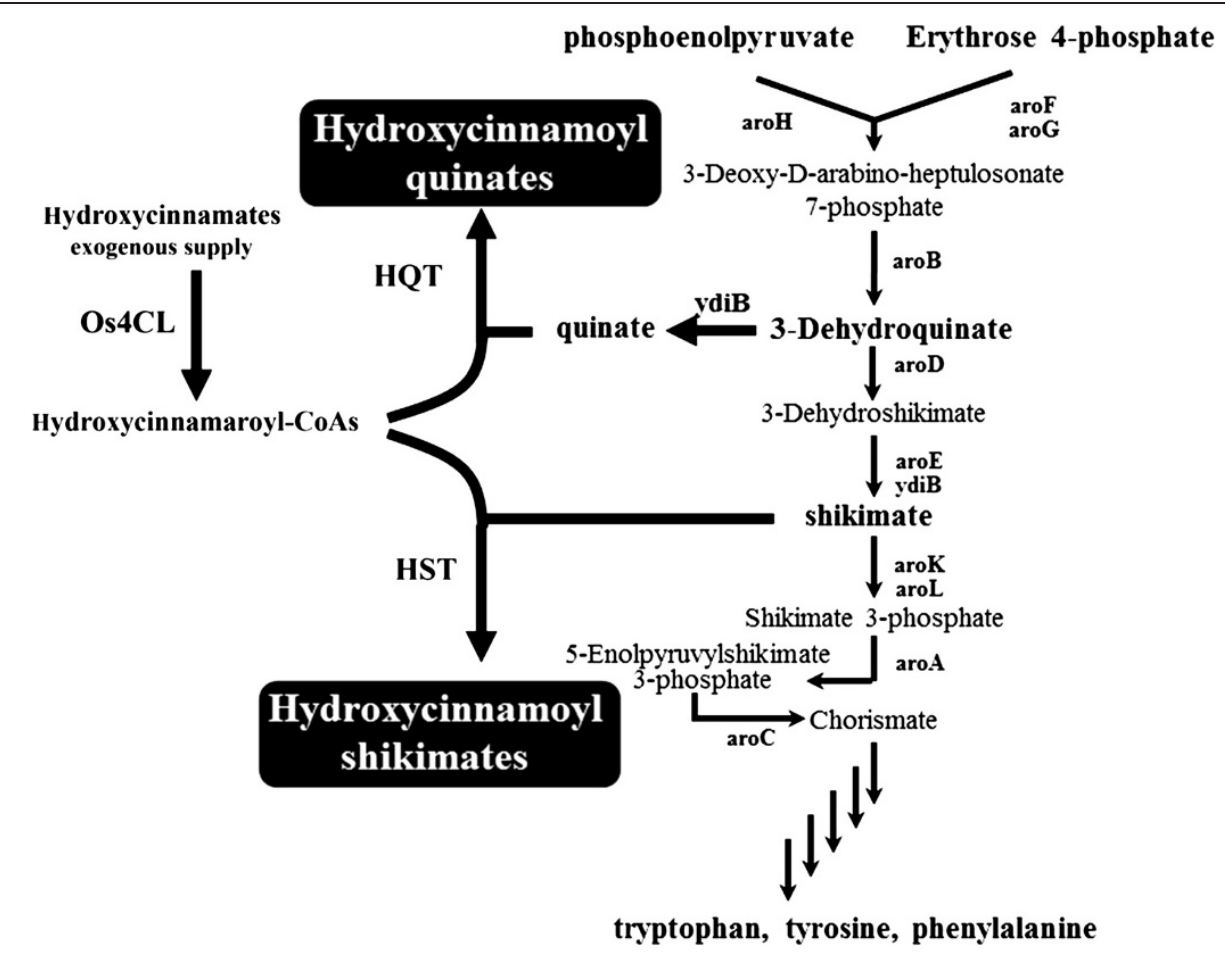

Figure 1 Schematic diagram showing the shikimate pathway of Escherichia coli and production of hydroxycinnamoyl quinate and hydroxycinnamoyl shikimate in E. coli.

shikimate molecule. $p$-Coumaroyl shikimates are not commercially available and the structures of the reaction products was determined using nuclear magnetic resonance spectroscopy (NMR). By the help of the HMBC and HMQC experiments (Additional file 1: Figure S1, $\mathrm{S} 2), 14{ }^{13} \mathrm{C}$ peaks were determined, so that $\mathrm{P} 1$ was considered to be a conjugate of PA and shikimate. Even this structure contains 16 carbons, two set symmetric carbons of PA provide only two carbon peaks. Based on the HMBC spectrum (Additional file 1: Figure S1), the carboxyl group of PA was connected to the hydroxyl group of shikimate. Three isomers, the carboxyl group of PA connected to $3 "-\mathrm{OH}$ or $4 "-\mathrm{OH}$ or 5 "-OH of shikimate, were possible. In the current NMR experiments, we could not determine the connected position. The name of the product P1 is (E)-3,4-dihydroxy-5-(3-(4-hydroxyphenyl)acryloyloxy)cyclohex-1-enecarboxylic acid where the bold number 5 could be switched with 3 or 4 .

Based on the molecular masses of P2, P3, and P4 (Figure 2C), they contained two PAs and one shikimate. Two PAs can be connected to 3"-OH or 4"-OH or 5"$\mathrm{OH}$ of $\mathrm{SA}$, so that there can be three isomers, i.e. 3"$\mathrm{OH} / 4$ "-OH, 3"-OH/5"-OH, or 4"-OH/5"-OH. We could not determine which produce was which isomer. By comparison to a previous report [21], P3 and P4 are likely to be 3,5-di-p-coumaroyl shikimate and 4,5-di-p-coumaroyl shikimate, respectively. The remaining peak, P2 is therefore likely to be 3,4-di-p-coumaroyl shikimate.
Using E. coli harboring pC-NtHST-Os4CL, we determined the best acyl donor among CA, PA, and FA. In a previous study, recombinant NtHST protein most efficiently used caffeoyl-CoA as an acyl donor [9]. In this study, $1 \mathrm{mM}$ of each $\mathrm{HC}$ was added to the same number of cells, and biotransformation was performed for $6 \mathrm{~h}$. PA was the most effective acyl donor, producing 23.9 $\mathrm{mg} / \mathrm{L}$ of $p$-coumaroyl shikimate. The amounts of feruloyl shikimate and caffeoyl shikimate produced were 3.8 $\mathrm{mg} / \mathrm{L}$, and $3.1 \mathrm{mg} / \mathrm{L}$, respectively. Taken together, these results indicate that the highest amount of $\mathrm{HC}$ shikimate was obtained with pC-NtHST-Os4CL as the construct and PA as the substrate.

\section{Engineering $E$. coli to increase production of PA-shikimate} conjugates

Because PA was added to the E. coli culture as an acyl donor, it was not a limiting factor for the production of PA-shikimate conjugates. However, E. coli uses endogenous shikimate as an acyl group acceptor. Thus, limiting shikimate availability would cause a bottleneck in the production of PA-shikimate conjugate. Previous studies showed that deletion of aroK and/or aroL in E. coli results in increased shikimate accumulation [22-24]. We created an E. coli aroL deletion mutant (BaroL), an aroK deletion mutant (BaroK) and an aroK/aroL double mutant (BaroKL) by gene replacement. pC-NtHST-Os4CL was transformed into each mutant and into wild type. 
Table 1 Plasmids, Escherichia coli strains, and primers used in this study

\begin{tabular}{|c|c|c|}
\hline Plasmids or E. coli strains or Primers & Relevant properties or genetic marker & Source or reference \\
\hline \multicolumn{3}{|l|}{ Plasmids } \\
\hline pCYCDuet & P15A ori, $\mathrm{Cm}^{\mathrm{r}}$ & Novagen \\
\hline pCDFDuet & CloDE13 ori, Str & Novagen \\
\hline pETDuet & f1 ori, Ampr & Novagen \\
\hline pA-NtHST-Os4CL & pACYCDDuet carrying NtHST from N. tobacco and $4 C L$ from O. sativa & This study \\
\hline pC-NtHST-Os4CL & pCDFDuet carrying NtHST from N. tobacco and $4 C L$ from O. sativa & This study \\
\hline pE-NtHST-Os4CL & pETDuet carrying NtHST from N. tobacco and $4 C L$ from $O$. sativa & This study \\
\hline pC-NtHQT-Os4CL & pCDFDuet carrying NtHQT from N. tobacco and $4 C L$ from O. sativa & This study \\
\hline pA-EcydiB & pACYC carrying ydiB from E. coli & This study \\
\hline \multicolumn{3}{|l|}{ Strains } \\
\hline BL21 (DE3) & $\mathrm{F}^{-}$ompT hsdS ${ }_{B}\left(\mathrm{r}_{\mathrm{B}^{-}} \mathrm{m}_{\mathrm{B}^{-}}\right)$gal dcm lon (DE3) & Novagen \\
\hline B-100 & BaroD carrying pCDF-Duet & This study \\
\hline B-101 & BaroD carrying pC-NtHQT-Os4CL & This study \\
\hline B-102 & BaroD carrying pC-NtHQT-Os $4 \mathrm{CL}$ and pA-EcydiB & This study \\
\hline BaroD & BL21(DE3) $\triangle a r o D: F R T-k a n^{R}-F R T$ & This study \\
\hline BaroK & BL21(DE3) $\triangle a r o K: F R T-k a n^{R}-F R T$ & This study \\
\hline BaroL & BL21(DE3) $\Delta a r o L:: F R T-k a n^{R}-F R T$ & This study \\
\hline BaroKL & BL21(DE3) $\triangle a$ aroK::FRT $\triangle a r o L:: F R T-k_{a n}{ }^{R}$-FRT & This study \\
\hline \multicolumn{3}{|l|}{ Primers } \\
\hline NtHST-F ${ }^{1}$ & \multicolumn{2}{|l|}{ AACATATGAAGATCGAAGTGAAAGAAT (Ndel site is underlined) } \\
\hline NtHST-R ${ }^{2}$ & \multicolumn{2}{|l|}{ AACTCGAGTCAAAAGTCATACAAGAACTTC (Xhol site is underlined) } \\
\hline NtHQT-F & \multicolumn{2}{|l|}{ AAGATATCCATGGGAAGTGAAAAAATGATGA (EcoRV site is underlined) } \\
\hline NtHQT-R & \multicolumn{2}{|l|}{ AAGGTACCTCAAAATTCATACAAATACTT (Kpnl site is underlined) } \\
\hline ydiB-F & \multicolumn{2}{|l|}{ ATGAATTCGATGGATGTTACCGCAAAATAC (ECORI site is underlined) } \\
\hline ydiB-R & \multicolumn{2}{|l|}{ CATGCGGCCGCTCAGGCACCGAACCCCATG (Notl site is underlined) } \\
\hline aroK-F & \multicolumn{2}{|l|}{ gctgtcttttttacgctaatcttacccggtgatttatcgccagagcggtgaattaaccctcactaaagggcg } \\
\hline aroK-R & \multicolumn{2}{|l|}{ cccgcagacgagtgtatataaagccagaattagttgctttccagcatgtgtaatacgactcactatagggctc } \\
\hline aroL-F & \multicolumn{2}{|c|}{ atgacaccggctttcgccgcattgcgacctattggggaaaacccacgatgaattaaccctcactaaagggcg } \\
\hline aroL-R & \multicolumn{2}{|l|}{ gatgaacgttaagtataggcgctcgaaaatcaacaattgatcgtctgtgctaatacgactcactatagggctc } \\
\hline aroD-F & \multicolumn{2}{|c|}{ tggggttcggtgcctgacaggctgaccgcgtgcagaaagggtaaaaaatgaattaaccctcactaaagggcg } \\
\hline aroD-R & \multicolumn{2}{|c|}{ gggaggatattcccgccgaaatattattgcttatgcctgatgtaaaatagttaatacgactcactatagggctc } \\
\hline aroK-check-F & \multicolumn{2}{|l|}{ cgctgcctgcgttccatgat } \\
\hline aroL-check-F & \multicolumn{2}{|l|}{ cgcggagctggagaagtggt } \\
\hline aroK or aroL check-R & \multicolumn{2}{|l|}{ taatacgactcactatagggctc } \\
\hline aroD-check-F & \multicolumn{2}{|l|}{ ggcaaggggctgaacagttc } \\
\hline aroD-check-R & \multicolumn{2}{|l|}{ gggaggatattcccgccgaa } \\
\hline
\end{tabular}

${ }^{1} \mathrm{~F}$ means forward primer.

${ }^{2} \mathrm{R}$ means reverse primer.

BaroL showed the highest productivity (approximately $235 \mathrm{mg} / \mathrm{L}$ ), which is approximately 15-fold higher than BL21 (16 mg/L). BaroKL and BaroK produced $228 \mathrm{mg} / \mathrm{L}$ and $171 \mathrm{mg} / \mathrm{L}$ of PA-shikimates, respectively (Figure 3). According to ANOVA test, PA-shikimates production was significantly different at $\mathrm{P}=0.01$ level among BL21,
BaroK, and BaroL. However, there was no difference between BaroL and BaroKL at this level of significance.

Next, production of PA-shikimate was optimized using BaroL harboring pC-NtHST-Os4CL. The optimum cell concentration was determined by varying the cell concentration from $\mathrm{OD}_{600} 1$ to 5 in the presence of $1 \mathrm{mM}$ 

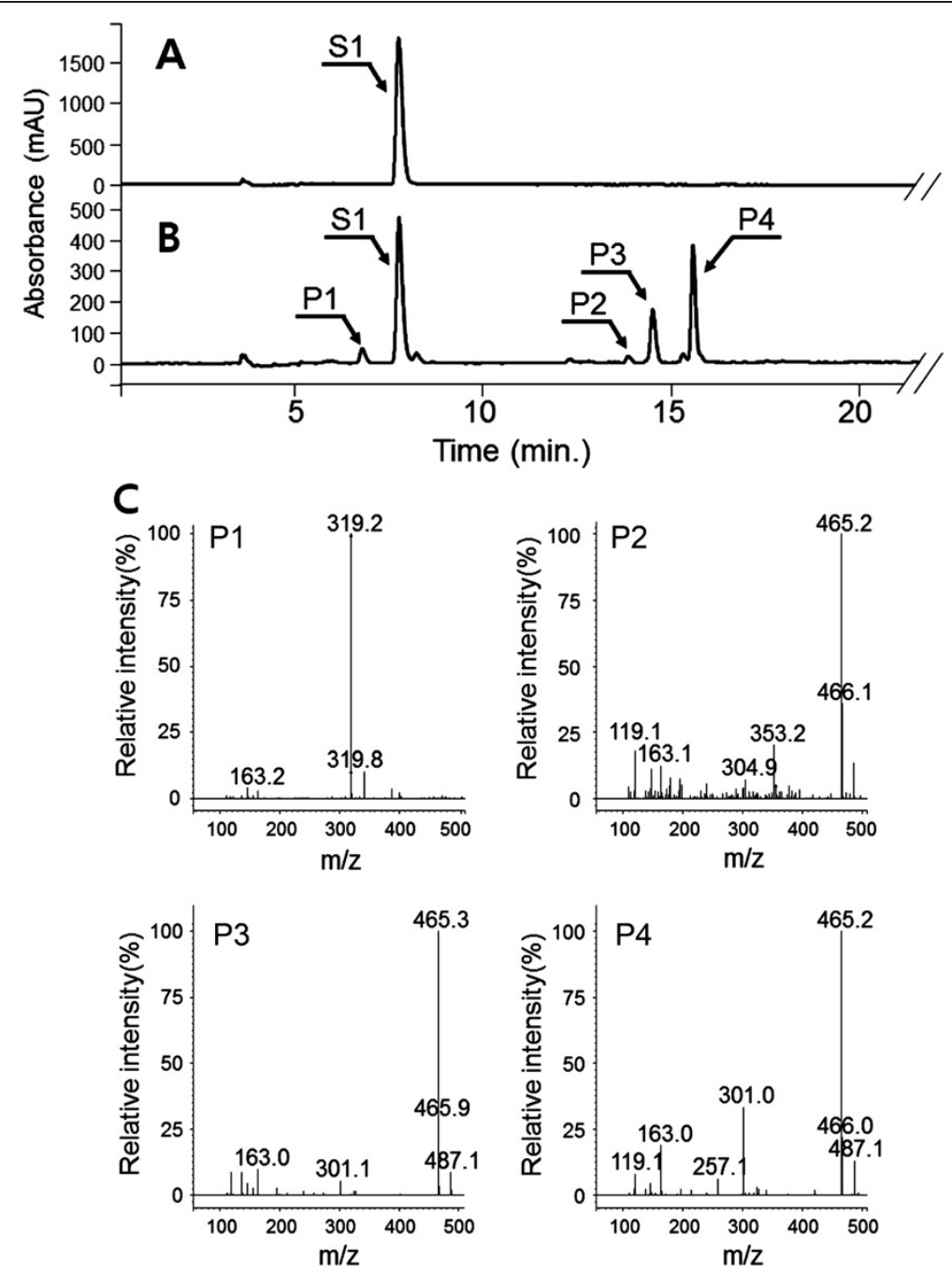

Figure 2 High performance liquid chromatography analysis of biotransformation of $p$-coumaric acid in BL21 (DE3) harboring Os4CL and NtHST. A, p-coumaric acid (S1); B, reaction products of p-coumaric acid (P1 - P4); C, mass spectrometry (MS) data for the four reaction products. The mass spectrometer was operated in negative mode. The molecular masses of $p$-coumaric acid and shikimic acid are 164 Da and $174 \mathrm{Da}$, respectively.

PA. Production of PA-shikimate peaked at an $\mathrm{OD}_{600}$ of 3 , at which cell concentration the yield of PA-shikimate was approximately $235 \mathrm{mg} / \mathrm{L}$. At $\mathrm{OD}_{600}=1$, or 2 , the yield was approximately $94 \mathrm{mg} / \mathrm{L}$, and $169 \mathrm{mg} / \mathrm{L}$, respectively. Above $\mathrm{OD}_{600}=3$, the production of PAshikimate decreased and was approximately 188 or 103.7 $\mathrm{mg} / \mathrm{L}$ at $\mathrm{OD}_{600}=4$ or 5 , respectively.

Using BaroL harboring pC-NtHST-Os4CL at a cell concentration of $\mathrm{OD}_{600} 3$, we monitored the production of HC-shikimate over $12 \mathrm{~h}$. The maximum amount of di-p-coumaroyl shikimates was produced at $8 \mathrm{~h}$ (134 $\mathrm{mg} / \mathrm{L}$ 3,5-di-p-coumaroyl shikimate, $70 \mathrm{mg} / \mathrm{L}$ 4,5-di-pcoumaroyl shikimate, $8 \mathrm{mg} / \mathrm{L}$ of 3,4-di-p-coumaroyl shikimate, and $23 \mathrm{mg} / \mathrm{L}$ of 5 -p-coumaroyl shikimate). Therefore, a total of $235 \mathrm{mg} / \mathrm{L}$ of PA-shikimates was produced. At $8 \mathrm{~h}$, the amount of 4,5-di-p-coumaroyl shikimate and 3,5-di-p-coumaroyl shikimate was reduced, but the amount of 5-p-coumaroyl shikimate reached a maximum (Figure 4). It seems that 5-p-coumaroyl shikimate was immediately converted to either 3,5-di- $p$-coumaroyl shikimate or 4,5-di- $p$-coumaroyl shikimate before $8 \mathrm{~h}$.

\section{Production of HC-quinate in E. coli}

We used an acyltransferase that is specific for quinate to produce HC-quinate. Hydroxycinnamoyl-CoA quinate transferase from $N$. tobacco (NtHQT) was shown to be more specific for quinate than for shikimate $[9,25]$. Os4CL mediates formation of coenzyme A thioester with $\mathrm{HC}$. To produce $\mathrm{HC}$-quinate in $E$. coli, two genes, $N t H Q T$ and Os4CL, were subcloned into an E. coli expression vector. The resulting construct, pC-NTHQT- 


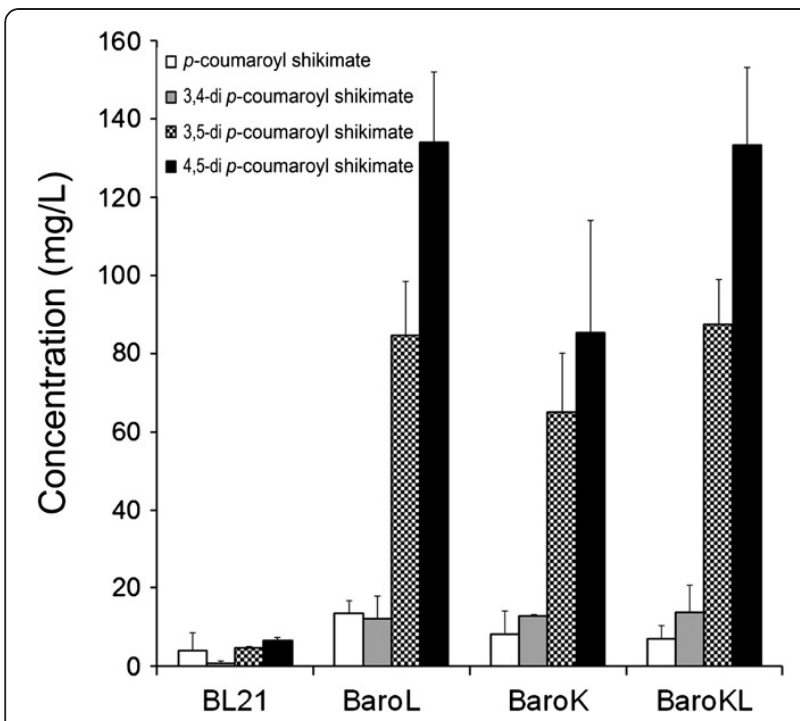

Figure 3 Production of hydroxycinnamic shikimates in different E. coli strains. Product 1 contains one molecule of hydroxycinnamate attached to shikimate, while products $2-4$ contain two molecules of hydroxycinnamate attached to shikimate. Error bars indicate mean values \pm SD from three independent experiments. Product 1 is 3 or 4 - $p$-coumaroyl shikimate; The structures of product 2 , product 3 , and product 4 are likely to be 3,4-di-p-coumaroyl 3,5-di-p-coumaroyl shikimate, and 4,5-di-p-coumaroyl shikimate, respectively.

Os4CL was transformed into BL21(DE3), and the transformant was used for biotransformation of CA. However, no reaction product was detectable. The amount of quinate or dehydroquinate in $E$. coli may not be high enough to drive the production of chlorogenic acid. Therefore, we created strain BaroD, which should accumulate higher levels of dehydroquinate and/or quinate, in order to overcome this potential limitation. The strain BaroD was used as a host for pC-NTHQT-Os4CL and the resulting transformant was called B-101 (Table 1). As control, empty pCDFDuet was transformed into the strain BaroD and this transformant was called B-100 (Table 1). Each transformant was used for biotransformation of CA. The culture medium of B100 became a brown color after $8 \mathrm{~h}$. Analysis of the reaction mixture using HPLC after $8 \mathrm{~h}$ incubation showed a trace level of caffeic acid. In addition, no detectable new product was observed. The decrease of caffeic acid during biotransformation is associated with the formation of $\mathrm{O}$-quinone and its polyaromatic derivatives [26]. However, analysis of the biotransformation mixture from strain B-101 showed a new peak (Figure 5C) and the color of the culture medium did not change. Once caffeic acid is converted into another compound, it cannot polymerize and therefore the color of the culture medium does not change. The molecular mass of the product was $352 \mathrm{Da}$, which was $2 \mathrm{Da}$ less than the predicted molecular mass of the thioester of quinate and CA (Figure 5C and E). Based on the molecular weight of the reaction product and the known intermediates of the $E$. coli shikimate pathway, it is like that 3-dehydroquinate and not quinate was attached to CA. NtHQT utilized 3-dehydroquinate as an acceptor and caffeoyl-CoA as a donor to make caffeoyl-3-dehydroquinate. Expression of $y d i B$ from E. coli, which converts dehydroquinate into quinate, also increased the production of $\mathrm{HC}$ ester yield because NtHQT was likely to have higher affinity for quinate than for dehydroquinate as acyl group acceptor.

The protein product of $y d i B$ converts 3-dehydroquinate into quinate [27]. $y d i B$ was overexpressed in E. coli strain

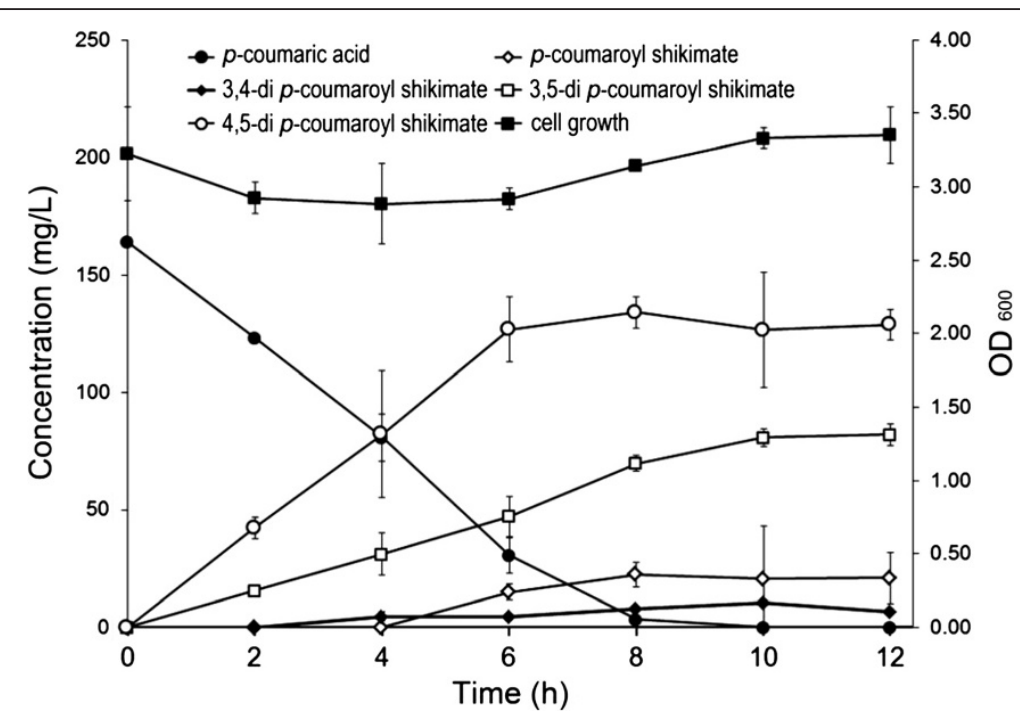

Figure 4 Production of hydroxycinnamic shikimates by biotransformation using BaroL harboring pC-NtHST-Os4CL. Error bars indicate mean values \pm SD from three independent experiments. 

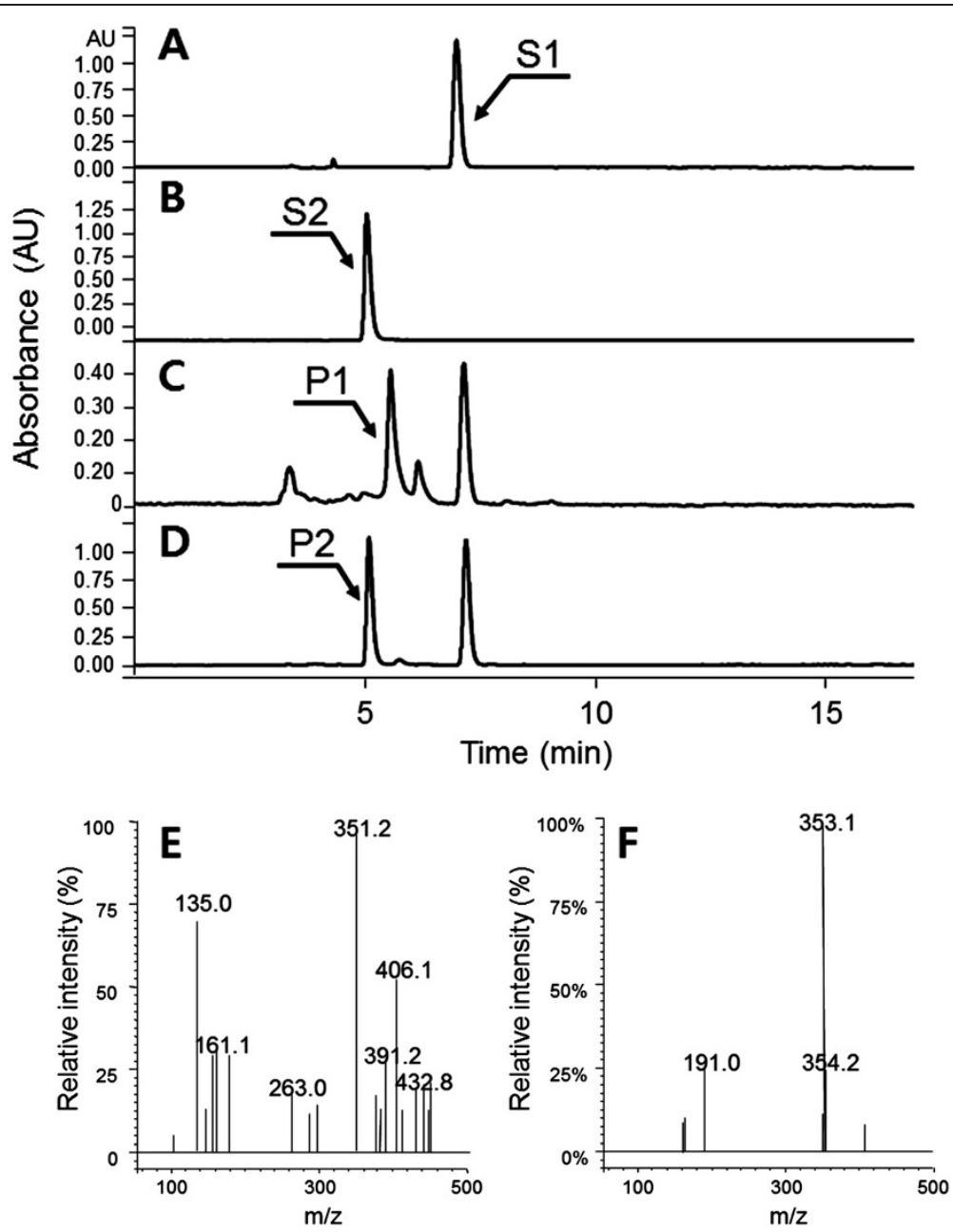

Figure 5 Production of chlorogenic acid in E. coli. A, caffeic acid (S1); B, chlorogenic acid (S2); C, reaction product of caffeic acid obtained from strain B-101; D, reaction product of caffeic acid obtained from strain B-102; E, MS/MS profile of P1; F, MS/MS profile of P2.

B-101 to make strain B-102. Biotransformation of CA by strain B-102 resulted in a new product with an identical HPLC retention time and molecular mass (354 Da) as chlorogenic acid (Figure 5D, F). The MS/MS spectrum of the reaction product matched authentic chlorogenic acid. This indicates that ydiB converts 3-dehydroquinate into quinate, which was then utilized for the production of chlorogenic acid.

PA and FA were also tested as acyl-group donors. CA was the best acyl-group donor followed by PA and FA. After $24 \mathrm{~h}$, the amounts of caffeoyl-quinate, $p$-coumaroyl-quinate, and feruloyl-quinate were $450 \mathrm{mg} / \mathrm{L}, 323.7$ $\mathrm{mg} / \mathrm{L}$, and $216 \mathrm{mg} / \mathrm{L}$, respectively.

The production of chlorogenic acid was monitored further in strain B-102. After induction, 1.1, 1.3, 1.5, 1.7, or $2.0 \mathrm{mM}$ CA was added to the culture. After $24 \mathrm{~h}$, almost all of the CA was converted into chlorogenic acid in the reaction mixtures supplied with $1.1,1.3$, or $1.5 \mathrm{mM}$ of CA. However, at a concentration of 1.7 or $2.0 \mathrm{mM}$, some CA remained in the mixture, and even after a longer incubation time, the remaining caffeic acid was not converted into chlorogenic acid. However, the color of the mixture became dark brown due to the oxidation/polymerization of CA. Therefore, we monitored chlorogenic acid production at a CA concentration of $1.5 \mathrm{mM}$. As shown in Figure 6, production of chlorogenic acid continued to increase until $24 \mathrm{~h}$, while the CA content decreased. Approximately 450 $\mathrm{mg} / \mathrm{L}$ of chlorogenic acid was produced at $24 \mathrm{~h}$.

\section{Discussion}

Anthocyanins, flavonoids, stilbene, and other compounds of plant origins have been biologically synthesized using engineered E. coli strains [7,15]. Most of these efforts involved introduction of new genes with high activity into $E$. coli. In addition, cofactor supplementation and reducing equivalents have been enhanced to produce natural compounds using engineered $E$. coli $[6,28]$. The goal of this study was to produce PA- 
shikimate and chlorogenic acid in E. coli. We also engineered the shikimate pathway of $E$. coli to accumulate the acyl group acceptors, shikimate and quinate, which led to the increased production of PA-shikimates and chlorogenic acid.

During biotransformation, two PA molecules are attached to shikimate, whereas only one molecule of CA is attached to quinate. In plants, one molecule of CA is bound to one molecule of shikimate. However, diverse $\mathrm{HC}$-quinate conjugates, including those in which two or three identical HCs are bound to quinate, and two or three different $\mathrm{HCs}$ are bound to quinate, were found in coffee (Coffea robusta) and sweet potato (Ipomonea batatas) [21,29]. In these plants, the mono-esters are present as major components whereas di- and tri-esters are present as minor components. NtHST and NtHQT may have different enzymatic properties from HCTs from coffee or sweet potato because di-p-coumaroyl skimates and cholorgenic acid were synthesized using NtHST and NtHQT. One possible scenario is that the substrate binding pocket of NtHQT may be narrower than that of NtHST. Thus, HC-quinate may not fit into the substrate binding pocket of NtHQT for the second round of acyl transfer reaction. Longer incubation of the recombinant NtHST enzyme with PA-CoA and shikimate resulted in the production of di-PA-shikimate (data not shown). The production of di-PA-shikimate was also observed with HST from Populus euramericana [13]. Using the properties of NtHST, diverse HCshikimate conjugates could possibly be obtained and feeding of different concentrations of various HCs into the E. coli culture medium may result in production of various forms of $\mathrm{HC}$-shikimate conjugates. Any biological activity including anti-oxidant activity of di-PAshikimate compared to PA-shikimate has not been tested. However, 8-O-4-diferulic acid showed better antioxidant activity than ferulic acid [30].

p-Coumaric acid can be synthesized from tyrosine by the action of tyrosine ammonia lyase (TAL). Therefore, synthesis of $p$-coumaroyl-shikimate or chlorogenic acid from glucose might be possible if a gene econding TAL were expressed into E. coli harboring NtHST and Os4CL or into E. coli harboring NtHQT, Os4CL, ydiB, and Sam5 (Sam5 converts $p$-coumaric acid into caffeic acid [31]), respectively. However, it is expected that only a tiny amount of reaction product would be produced from glucose in a wild type strain because quinate and shikimate are not accumulated in the wild type E. coli. Use of strain BaroD or BaroL is not also feasible because tyrosine is not synthesized in these strains. Thus, supplementation with either caffeic acid or tyrosine is needed to synthesize $p$-coumaroyl-shikimate or chlorogenic acid in E. coli.

Wild type E. coli expressing NtHQT and Os4CL did not produce detectable amounts of chlorogenic acid or caffeoyl-dehydroquinate after CA supplementation. In the wild type, dehydroquinate may have been rapidly converted into another downstream compound of the shikimate pathway instead of accumulating. Moreover, the expression level of $y d i B$, which converts dehydroquinate into quinate, was low. Only a small amount of quinate or dehydroquinate will be expected to be present in the cell in the absence of overexpression of $y d i B$ [32]. $y d i B$ and aroE mediate the conversion reaction not only from dehydroquinate to quinate but also from dehydroshikimate to shikimate. However, aroE prefers dehydroshikimate to dehydroquinate [22], whereas ydiB has nearly the same catalytic efficiency for dehydroshikimate and dehydroquinate [27,33]. Recent studies have shown that overexpression of $y d i B$ does not increase shikimate,

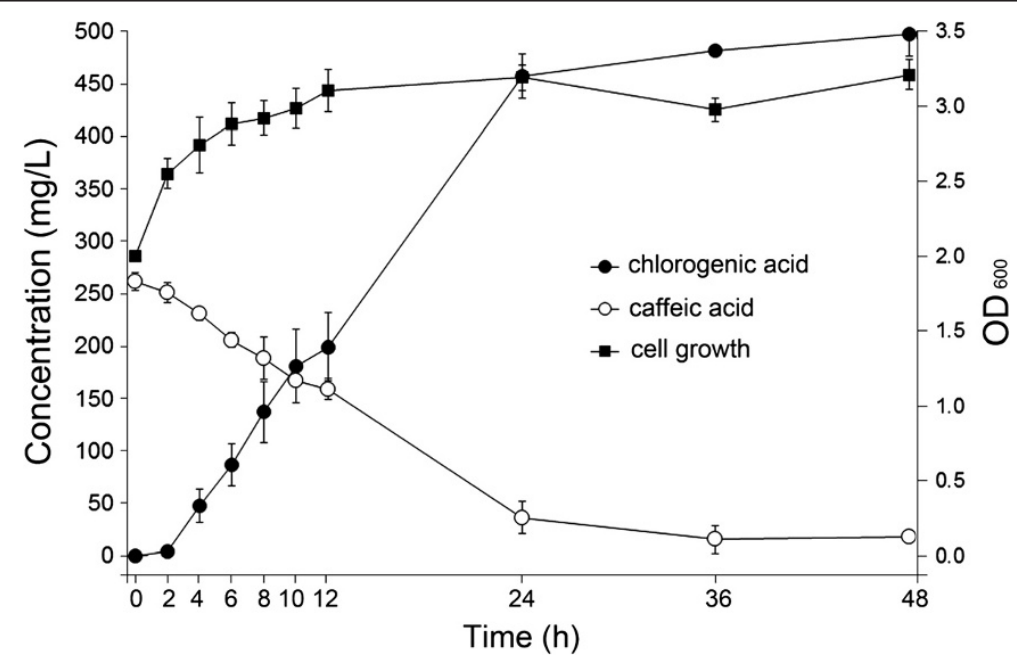

Figure 6 Production of chlorogenic acid using the E. coli strain B-106. 
while overexpression of aroE increases conversion of dehydroshikimate to shikimate [32]. Therefore, we overexpressed $y d i B$ to produce chlorogenic acid. In addition, strains BaroK, BaroL, and BaroKL expressing pC-NtHQT-Os4CL that were supplemented with CA produced chlorogenic acid only when ydiB was overexpressed (data not shown). This indicated that these mutants accumulate shikimate pathway intermediates such as dehydroquinate, dehydroshikimate and shikimate. These intermediates are converted into quiniate by ydiB.

\section{Conclusions}

To produce the HC-conjugates in E. coli, several E. coli mutants including aroD, aroK, aroL, and aroK/L mutants were made and each mutant was tested using an optimized construct. For the production of HC-shikimates, an E. coli aroL mutant (B-aroL) was best and we produced $235 \mathrm{mg} / \mathrm{L}$ of $\mathrm{HC}$-shikimates using $\mathrm{B}$-aroL expressing NtHST and Os4CL, which is approximately 15-fold higher than wild type E. coli BL21 (16 mg/L). The wild type E. coli expressing NtHQT and Os4CL did not produce any detectable chlorogenic acid. By using $E$. coli aroD mutant (strain B-101), which accumulated 3-dehydroquinate, caffeoyl-3-dehydroquinate instead of chlorogenic acid, was synthesized. However, by expressing $y d i B$ gene in strain B-101, which converted 3dehyroquinate to quinate, chlorogenic acid production was dramatically increased up to $450 \mathrm{mg} / \mathrm{L}$.

\section{Materials and methods}

\section{Strains and reagents}

The E. coli strains used in this study are listed in Table 1. E. coli BL21 (DE3) cells were used for recombinant protein production. E. coli $\mathrm{DH} 5 \alpha$ cells were used for plasmid cloning. All restriction enzymes and T4 DNA ligase were purchased from Takara (Shiga, Japan). Polymerase chain reaction (PCR) amplification was performed using Hotstart Taq DNA polymerase (Qiagen, Hilden Germany). Reverse transcription was performed using Omniscript reverse transcriptase (Qiagen). E. coli was cultured in Luria-Bertani (LB) or M9 medium (plus 2\% glucose) containing $50 \mu \mathrm{g} / \mathrm{mL}$ antibiotics, when necessary. E. coli expression vectors were purchased from Novagen (Madison, WI, USA).

\section{Construction of $E$. coli expression vector}

The genes for hydroxycinnamate-CoA shikimate transferase (NtHST) [9] and hydroxycinnamate-CoA quinate transferase (NtHQT) from tobacco ( $N$. tabacum) [26] were cloned using reverse-transcription polymerase chain reaction (RT-PCR). Total RNA was isolated from the leaves of one-month-old tobacco using Plant Total RNA Isolation Kit (Qiagen), and cDNA was synthesized using Omniscript reverse transcriptase (Qiagen) and oligo $\mathrm{dT}$ as a primer. PCR was carried out using primers designed on the basis of the published sequences (GenBank accession AJ507825 for NtHST and AJ582651 for NtHQT). The primers were listed in Table 1. The Os4CL gene, which was cloned and characterized previously from rice [19], was subcloned into the BamHI/ NotI sites of pACYCDuet, pCDFDuet, and pETDuet vectors (EMD Chemicals, Gibbstown, NJ, USA), and then the resulting NtHST PCR product was subcloned into a second cloning site, the $\mathrm{NdeI} / \mathrm{XhoI}$ site of each vector. The NtHQT PCR product was subcloned into the EcoRV/KpnI site of pCDFDuet, which contains Os4CL at NdeI/XhoI site. The resulting constructs are listed in Table 1.

Deletion of the aroL and aroK genes in E. coli BL21 (DE3) was accomplished using the Quick and Easy Conditional Knockout Kit (Gene Bridges, Heidelberg, Germany). Briefly, the aroL gene or the aroK gene of $E$. coli BL21 (DE3) was replaced by the $\Delta a r o L$ FRT-PGKgb2-neo-FRT cassette or the $\triangle$ aroK FRT-PGK-gb2-neoFRT cassette, respectively [34]. Deletion mutants were selected in Luria-Bertani (LB) medium containing $50 \mu \mathrm{g} /$ $\mathrm{mL}$ kanamycin. Deletion of aroL or aroK was confirmed using PCR. The strains deleted in aroL or aroK were named BaroL and BaroK, respectively (Table 1). $\Delta a r o L$ and $\triangle a r o K$ double mutant (strain BaroKL in Table 1) was constructed using strain BaroL. The kanamycin cassette was removed in $\triangle a r o L$ using an FLP expression plasmid, which removes the kanamycin selection marker from the chromosome. Removal of the kanamycin cassette was confirmed by PCR. Using $\triangle a r o L$ as a host, the aroK gene was replaced by the $\triangle a r o K$ FRT-PGK-gb2neo-FRT cassette. The primer sequences for the aroD, aroK, and aroL deletion are shown in Table 1.

Shikimate/quinate dehydrogenase gene, $y d i B$ was cloned using E. coli BL21 (DE3) genomic DNA as a template. Primers were designed based on the published sequence (NC_000913.2) and the sequences of primers were listed in Table 1. The PCR product was digested with EcoRI/NotI and subcloned into the corresponding site of pCDF-Duet1. The resulting constructs were confirmed by sequencing.

\section{Production of HC-shikimate conjugate in E. coli}

Each construct was transformed into E. coli BL21 (DE3) strain or BaroL strain using electroporation with the BioRad MicroPulser Electroporation Apparatus (BioRad, Hercules, CA, USA). Overnight cultures of transformants were inoculated into LB medium containing $50 \mu \mathrm{g} / \mathrm{mL}$ of antibiotic at $37^{\circ} \mathrm{C}$ and cultured until the $\mathrm{OD}_{600}$ reached 0.8 . Protein expression was induced by the addition of $1 \mathrm{mM}$ isopropyl $\beta$-D-1-thiogalactopyranoside (IPTG), and growth was continued for another $20 \mathrm{~h}$ at $18^{\circ} \mathrm{C}$. Cells were harvested and resuspended to obtain a cell concentration 
corresponding to $\mathrm{OD}_{600}$ of 3 in $2 \mathrm{~mL}$ of fresh M9 medium containing $2 \%$ glucose, $50 \mu \mathrm{g} / \mathrm{mL}$ of antibiotics, $1 \mathrm{mM}$ IPTG, and $1 \mathrm{mM}$ PA in a test tube $(14 \mathrm{~mm} \times 145 \mathrm{~mm})$ and then cultured at $30^{\circ} \mathrm{C}$ for $8 \mathrm{~h}$. The supernatant was extracted twice using an equal volume of ethyl acetate, and the upper aqueous phase was dried using a Speed Vac at $30^{\circ} \mathrm{C}$, dissolved in $60 \mu \mathrm{L}$ of dimethylsulfoxide (DMSO), and analyzed using high-performance liquid chromatography (HPLC). Because $p$-coumaroyl shikimate is not commercially available, we used $p$-coumaric acid to generate a standard curve for quantitative analysis of the reaction products. The UV spectra of $p$-coumaric acid are distinguishable from those of $p$-coumaroyl shikimate but they are very similar and the absorption of the thioesters is usually shifted to longer wavelength by a few nm. This is subtle enough that it should be perfectly acceptable to use the free acids as quantitation standards for the thioesters.

To determine the maximum conversion rate using the optimized vector, protein expression was induced as described above. The cell concentration was adjusted to an $\mathrm{OD}_{600}$ of 3 in $25 \mathrm{~mL}$ of fresh M9 containing 2\% glucose, $1 \mathrm{mM}$ IPTG, and $50 \mu \mathrm{g} / \mathrm{mL}$ of antibiotics. The medium was supplemented with $1 \mathrm{mM}$ PA. The reaction product $(200 \mu \mathrm{L})$ was collected and extracted with ethyl acetate. The supernatant was dried and dissolved with $100 \mu \mathrm{l}$ of dimethyl sulfoxide (DMSO). The sample was directly injected to HPLC for analysis. The mean and the standard error of the mean were calculated from triplicate experiments. Analysis of variance (ANOVA) was carried out using Tukey's method with a significance level of $\mathrm{P}=0.01$ using 2010 Microsoft Office Excel.

\section{Production of chlorogenic acid in E. coli}

The construct for the production of chlorogenic acid was transformed into E. coli BL21(DE3) or BaroD cells. Induction of each construct was performed as described above. To determine the optimal gene construct, $200 \mu \mathrm{M}$ PA was added to the growth medium.

To measure the production of chlorogenic acid in BaroD cells harboring pC-EcycdiB and pC-NtHQT-Os4CL (Table 1), the cell concentration was adjusted to an $\mathrm{OD}_{600}$ of 2.0, and CA was added to the medium to a final concentration of $1.5 \mathrm{mM}$. Production of chlorogenic acid was periodically monitored. The biotransformation was stopped by boiling for $5 \mathrm{~min}$ and the biotransformation product was centrifuged for $15 \mathrm{~min}$ at $13000 \times \mathrm{g}$ to remove the cell debris and other components prior to HPLC analysis. The quantification of the product was carried out using a standard curve generated with authentic chlorogenic acid (Sigma, MO, USA).

\section{Analysis of the metabolites}

The metabolites were analyzed using a Varian HPLC equipped with a photo diode array (PDA) detector and a
Varian C18 reversed-phase column (Varian, $4.60 \times 250$ $\mathrm{mm}, 3.5 \mu \mathrm{m}$ particle size). The mobile phases consisted of $0.1 \%$ formic acid in water and acetonitrile. For chlorogenic acid, the program was: $20 \%$ acetonitrile at $0 \mathrm{~min}$, $32 \%$ acetonitrile at $15 \mathrm{~min}, 90 \%$ acetonitrile at $17 \mathrm{~min}$, $90 \%$ acetonitrile at $20 \mathrm{~min}, 20 \%$ acetonitrile at $21 \mathrm{~min}$, and $20 \%$ acetonitrile at $26 \mathrm{~min}$. To analyze hydroxycinnamoyl shikimate, the program was: $25 \%$ acetonitrile at $0 \mathrm{~min}, 40 \%$ acetonitrile at $10 \mathrm{~min}, 75 \%$ acetonitrile at 15 min, $90 \%$ acetonitrile at $22 \mathrm{~min}, 25 \%$ acetonitrile at 23 $\mathrm{min}$, and $25 \%$ acetonitrile at $30 \mathrm{~min}$. The flow rate was 1 $\mathrm{mL} / \mathrm{min}$, and the separation was monitored at $290 \mathrm{~nm}$ and $320 \mathrm{~nm}$.

The molecular masses of the metabolites were determined using a Varian 500-MS ion trap spectrometer. Mass spectra were acquired simultaneously using an electrospray ionization source in negative ionization mode at $600 \mathrm{~V}$. NMR spectrometry was done as described before [35].

\section{Additional file}

Additional file 1: Figure S1. The HMBC spectrum of the product P1. Figure S2. The HMQC spectrum of the product P1.

Competing interests

The authors declare that they have no competing interests.

\section{Authors' contributions}

JHA initiated and coordinated the project. BGK, JWD, HM, and JHA performed experiments, analyzed data and wrote the paper. All authors approved the final manuscript.

\section{Acknowledgements}

This work was supported by a grant from Systems and Synthetic AgroBiotech Center through the Next-Generation BioGreen 21 Program (PJ00948301), Rural Development Administration, and partially funded by the Priority Research Centers Program through the National Research Foundation of Korea (NRF) funded by the Ministry of Education, Science and Technology (2012-0006686).

Received: 11 December 2012 Accepted: 2 February 2013

Published: 5 February 2013

\section{References}

1. El-Seedi HR, El-Seed AMA, Khalifa SAM, Görasson U, Bohlin L, Borg-Karlson A-K, Verpoorte R: Biosynthesis, natural sources, dietary intake, pharmacokinetic properties, and biological activities of hydroxycinnamic acids. J Agri Food Chem 2012, 60(44):10877-10895.

2. Clifford MN: Chlorogenic acids and other cinnamates-nature, occurrence and dietary burden. J Sci Food Agric 1999, 79(3):362-372.

3. Herrmann K: Occurrence and content of hydroxycinnamic acid and hydoxybenzoic acid compounds in foods. Crit Rev Food Sci Nutr 1989, 28(4):315-347.

4. Crozier A, Jaganatha IB, Clifford MM: Dietary phenolics: chemistry, bioavailability and effects on health. Nat Prod Rep 2009, 26(8):1001-10043.

5. Chemler JA, Koffas MAG: Metabolic engineering for plant natural product biosynthesis in microbes. Cur Opin Biotech 2008, 19(6):597-605.

6. Chemler JA, Fowler ZL, Mchugh KP, Koffas MAG: Improving NADPH availability for natural product biosynthesis in Escherichia coli by metabolic engineering. Metab Eng 2010, 12(2):96-104. 
7. Fowler ZL, Koffas MAG: Biosynthesis and biotechnological production of flavanones: current state and perspectives. Appl Microbiol Biotechnol 2009, 83(5):799-808

8. Beuerle T, Pichershy E: Enzymatic synthesis and purification of aromatic coenzyme A esters. Anal Biochem 2002, 302(2):305-312.

9. Hoffmann L, Maury S, Martz F, Geoffroy P, Legrand M: Purification, cloning, and properties of an acyltransferase controlling shikimate and quinate ester intermediates in phenylpropanoid metabolism. J Biol Chem 2003, 278(1):95-103.

10. Hoffmann L, Besseau S, Geoffoy P, Ritzenthaler C, Meyer D, Lapierre C, Pollet B, Legrand M: Silencing of hydroxycinnamoyl-Coenzyme A shikimate/quinate hydroxycinnamoyltransferase affects phenylpropanoid biosynthesis. Plant Cell 2004, 16(6):1446-1465.

11. Wagner A, Ralph J, Akiyama T, Flint H, Phillips L, Torr K, Nanayakkara B, Kiri $\mathrm{LT}$ : Exploring lignification in conifers by silencing hydroxycinnamoyl-CoA: shikimate hydroxycinnamoyltransferase in Pinus radiata. Proc Natl Aca Sci USA 2007, 104(28):11856-11861.

12. Comino C, Lanteri S, Portis E, Acquadro A, Romani A, Hehn A, Larbat R, Bourgaud F: Isolation and functional characterization of a cDNA coding a hydroxycinnamoyltransferase involved in phenylpropanoid biosynthesis in Cynara cardunculus L. BMC Plant Biol 2007, 20(7):14

13. Kim B-G, Lee ET, Ahn J-H: Characterization of hydroxycinnamoylcoenzyme A shikimate hydroxycinnamoyltransferase from Populus euramericana. J Kor Soc Appl Biol Chem 2011, 54(2):817-821.

14. St Pierre B, De Luca V: Evolution of acyltransferase genes: origin and diversification of the BAHD superfamily of acyltransferases involved in secondary metabolism. In Recent Advances in Phytochemistry Vol 34. Evolution of Metabolic Pathways. Edited by Romeo JT, Ibrahim R, Varin L, De Luca V. Oxford: Elsevier Science Ltd; 2000:285-315.

15. Horinouchi S: Combinatorial biosynthesis of non-bacterial and unnatural flavonoids, stilbenoids and curcuminoids by microorganisms. J Antibiot 2008, 6(12):709-728.

16. Ikeda M: Towards bacterial strains overproducing L-tryptophan and other aromatics by metabolic engineering. Appl Microbiol Biotechnol 2006, 69(6):615-626

17. Gosset G: Production of aromatic compounds in bacteria. Curr Opin Biotech 2009, 20(6):651-658

18. Dixon RA, Paiva NL: Stress-induced phenylpropanoid metabolism. Plant Cell 1995, 7(7):1085-1097.

19. LeeY J, Jeon Y, Lee JS, Kim B-G, Lee CH, Ahn J-H: Enzymatic synthesis of phenolic CoAs using 4-coumarate:coenzyme $A$ ligase $(4 \mathrm{CL})$ from rice. Bull Kor Chem Soc 2007, 28(3):365-366.

20. Diaz E, Ferrández A, Prieto MA, Garcia JL: Biodegradation of aromatic compounds by Escherichia coli. Microbiol Mol Biol Rev 2001, 65(4):523-569.

21. Jaiswal R, Patras MA, Eravuchira PJ, Kuhnert N: Profile and characterization of the chlorogenic acids in green robusta coffee beans by LC-MS $^{n}$ : Identification of seven new classes of compounds. J Agric Food Chem 2010, 58(15):8722-8737.

22. Draths KM, Knop DR, Frost JW: Shikimic acid and quinic acid: replacing isolation from plant sources with recombinant microbial biocatalysis. J Am Chem Soc 1999, 121(7):1603-1604.

23. Escalante A, Calderón R, Valdivia A, de Anda R, Hernánde G, Ramirez OT, Gosset G, Bolivar F: Metabolic engineering for the production of shikimic acid in an evolved Esherichia coli strain lacking the phosphoenolpyruvate: carbohydrate phosphotransferase system. Microbial Cell Fact 2010, 9:21-33.

24. Krämer M, Bongaerts J, Bovenberg R, Kremer S, Müller U, Orf S, Wubbolts M, Raeven L: Metabolic engineering for microbial production of shikimic acid. Met Eng 2003, 5(4):277-283.

25. Niggeweg R, Michael AJ, Martin C: Engineering plants with increased levels of the antioxidant chlorogenic acid. Nat Biotech 2004, 22(4):746-754

26. Zhang H, Stephanopoulos G: Engineering E. coli for caffeic acid biosynthesis from renewable sugar. Appl Microbiol Biotechnol 2012 doi:10.1007/s00253-012-4544-8.

27. Lindner HA, Nadeau G, Matte A, Michel G, Ménard R, Cygler M: Site-directed mutagenesis of the active site region in the quinate/ shimate 5-dehydrogenase YdiB of Escherichia coli. J Biol Chem 2005, 280(8):7162-7169.

28. Sung $\mathrm{SH}, \mathrm{Kim}$ BG, Ahn J-H: Optimization of rhamnetin production in Escherichia coli. J Microbio Biotech 2011, 21(8):854-857.
29. Zheng W, Clifford MN: Profiling the chlorogenic acids of sweet potato (Ipomoea batatas) from China. Food Chem 2010, 106(1):147-152.

30. Garcia-Conesa MT, Plumb GW, Waldron KW, Ralph J, Williamson G: Ferulic acid dehydrodimers from wheat bran: isolation, purification and antioxidant properties of 8-O-4-diferulic acid. Redox Rep 1997, 3(5-6):319-323.

31. Berner M, Krug D, Bihlmaier C, Vente A, Müller R, Bechthold A: Genes and enzymes involved in caffeic acid biosynthesis in the actinomycete Saccharothix espanaensis. J Bact 2006, 188(7):2666-2673.

32. Juminaga D, Baidoo EE, Redding-Johanson AM, Batth TS, Burd H, Mukhopadhyay A, Petzold CJ, Keasling JD: Modular engineering of Ltyrosine production in Escherichia coli. Appl Environ Microbiol 2012, 78(1):89-98.

33. Michel G, Roszak AW, Sauvé V, Maclean J, Matte A, Coggins JR, Cygler M, Lapthorn AJ: Structure of shikimate dehydrogenase AroE and its paralog YdiB. J Biol Chem 2003, 278(21):19463-19472.

34. Datsenko KA, Wanner BL: One-step inactivation of chromosomal genes in Escherichia coli K-12 using PCR products. Proc Natl Acad Sci USA 2000, 97(12):6640-6645.

35. Yoon J-A, Kim B-G, Lee WJ, Lim Y, Chong Y, Ahn J-H: Production of a novel quercetin glycoside through metabolic engineering of Escherichia coli. Appl Env Microbiol 2012, 78(12):4256-4262.

doi:10.1186/1475-2859-12-15

Cite this article as: Kim et al:: Production of hydroxycinnamoyl-

shikimates and chlorogenic acid in Escherichia coli: production of hydroxycinnamic acid conjugates. Microbial Cell Factories 2013 12:15.

\section{Submit your next manuscript to BioMed Central and take full advantage of:}

- Convenient online submission

- Thorough peer review

- No space constraints or color figure charges

- Immediate publication on acceptance

- Inclusion in PubMed, CAS, Scopus and Google Scholar

- Research which is freely available for redistribution
C Biomed Central 